\title{
Statistics of extremes in eigenvalue-counting staircases
}

\author{
Yan V. Fyodorov ${ }^{1,2}$ and Pierre Le Doussal ${ }^{3}$ \\ ${ }^{1}$ King's College London, Department of Mathematics, London WC2R 2LS, United Kingdom \\ ${ }^{2}$ L.D.Landau Institute for Theoretical Physics, Semenova 1a, 142432 Chernogolovka, Russia \\ ${ }^{3}$ Laboratoire de Physique Théorique de l'Ecole Normale Supérieure, PSL Research University, \\ CNRS, Sorbonne Universités, 24 rue Lhomond, 75231 Paris, France
}

\begin{abstract}
We consider the number $\mathcal{N}_{\theta_{A}}(\theta)$ of eigenvalues $e^{i \theta_{j}}$ of a random unitary matrix, drawn from $\operatorname{CUE}_{\beta}(N)$, in the interval $\theta_{j} \in\left[\theta_{A}, \theta\right]$. The deviations from its mean, $\mathcal{N}_{\theta_{A}}(\theta)-\mathbb{E}\left(\mathcal{N}_{\theta_{A}}(\theta)\right)$, form a random process as function of $\theta$. We study the maximum of this process, by exploiting the mapping onto the statistical mechanics of log-correlated random landscapes. By using an extended FisherHartwig conjecture supplemented with the freezing duality conjecture for log-correlated fields, we obtain the cumulants of the distribution of that maximum for any $\beta>0$. It exhibits combined features of standard counting statistics of fermions (free for $\beta=2$ and with Sutherland-type interaction for $\beta \neq 2$ ) in an interval and extremal statistics of the fractional Brownian motion with Hurst index $H=0$. The $\beta=2$ results are expected to apply to the statistics of zeroes of the Riemann Zeta function.
\end{abstract}

PACS numbers:

Characterizing the full counting statistics of the fluctuations of the number $\mathcal{N}$ of $1 d$ fermions in an interval is important in numerous physical contexts, both for ground state and dynamical properties. It appears e.g. in shot noise [1, in fermion chains 2, 3, in interacting Bose gases 4], in non-equilibrium Luttinger liquids [5], in trapped fermions [6] servables, such as the entanglement entropy [9 11] or the statistics of local magnetization in quantum spin chains [12]. An equivalent problem can be formulated as counting eigenvalues of large random matrices (RM). As is well known since Dyson's work [13, such eigenvalues behave as classical particles with 1-d Coulomb repulsion at inverse temperature $\beta>0$. Namely, consider a unitary $N \times N$ matrix $U$ and denote the corresponding unimodular eigenvalues as $z_{j}=e^{i \theta_{j}}, j=1, \ldots, N$, with phases $\left.\left.\theta_{i} \in\right]-\pi, \pi\right]$. Then for any given $\beta>0$ one can construct the so-called Circular $\beta$-Ensemble $\operatorname{CUE}_{\beta}(N)$ in such a way that the expectation of a function depending only on the eigenvalues of $U$ will be given by

$$
\mathbb{E}(F)=c_{N} \prod_{j=1}^{N} \int_{-\pi}^{\pi} d \theta_{i} \prod_{1 \leq j<k \leq N}\left|e^{i \theta_{j}}-e^{i \theta_{k}}\right|^{\beta} F
$$

where $F \equiv F\left(\theta_{1}, \ldots, \theta_{n}\right)$. For $\beta=2$ such matrices can be thought of as drawn uniformly according to the corresponding Haar's measure on $U(N)$, whereas for a generic $\beta>0$ the explicit construction is more involved, see [14. For any $\beta>1$, the r.h.s of (1) equals the quantum expectation value of $F$ in the ground state of $N$ spinless fermions, of coordinates $\theta_{i}$ on the unit circle, described by the Sutherland Hamiltonian [15] $H=$ $-\sum_{i} \frac{\partial^{2}}{\partial \theta_{i}^{2}}+\sum_{i<j} \frac{\beta(\beta-2)}{8 \sin ^{2}\left(\frac{\theta_{i}-\theta_{j}}{2}\right)}$. For $\beta=2$, Eq (1) thus describes non-interacting fermions, while for $\beta \neq 2$ the fermions interact, via an inverse square distance pairwise potential.
Let us now define the number of eigenvalues/fermions, $\mathcal{N}_{\theta_{A}}(\theta)$, in the interval $\theta_{j} \in\left[\theta_{A}, \theta\right]$ as

$\mathcal{N}_{\theta_{A}}(\theta)=\sum_{j=1}^{N}\left(\chi\left(\theta-\theta_{j}\right)-\chi\left(\theta_{A}-\theta_{j}\right)\right), \chi(u)= \begin{cases}1, & u>0 \\ 0, & u<0\end{cases}$

As a function of $\theta$ this is a staircase with unit jumps upwards at random positions $\theta_{j} \in\left[\theta_{A}, \theta\right]$. The mean slope (i.e. the mean density of eigenvalues/fermions) being constant, the mean profile is $\mathbb{E}\left(\mathcal{N}_{\theta_{A}}(\theta)\right)=\frac{N\left(\theta-\theta_{A}\right)}{2 \pi}$. In a given random matrix realization/sample one can define the deviation to the mean, $\delta \mathcal{N}_{\theta_{A}}(\theta)=\mathcal{N}_{\theta_{A}}(\theta)-$ $\mathbb{E}\left(\mathcal{N}_{\theta_{A}}(\theta)\right)$, and study it as a random process as a function of $\theta$, i.e. as a function of the length of the interval $\theta-\theta_{A}$, see Fig. 1 and 2 . From the view of such a process, the standard results on fermion counting statistics [2], encoding the full distribution of $\delta \mathcal{N}_{\theta_{A}}(\theta)$ for a fixed value of $\theta$, is a very local information. Such information is clearly insufficient for understanding various non-local properties of the process, such as characterizing maximal deviation of the staircase from its mean, i.e. $\max _{\theta \in\left[\theta_{A}, \theta_{B}\right]}\left|\mathcal{N}_{\theta_{A}}(\theta)-\mathbb{E}\left(\mathcal{N}_{\theta_{A}}(\theta)\right)\right|$. After normalization this is the Kolmogorov-Smirnov (KS) statistics, an outstanding open problem for spectra of random matrices [16], [17].

In this Letter we study the value distribution separately for the maximum (and equivalently the minimum) of the centered process by explicitly calculating the $\mathrm{cu}-$ mulants of the probability density function (PDF) for the maximum value defined as

$$
\delta \mathcal{N}_{m}=\max _{\theta \in\left[\theta_{A}, \theta_{B}\right]}\left\{\mathcal{N}_{\theta_{A}}(\theta)-\mathbb{E}\left(\mathcal{N}_{\theta_{A}}(\theta)\right)\right\}
$$

on an interval $\left.\left.\left[\theta_{A}, \theta_{B}\right] \subset\right]-\pi, \pi\right]$, of a fixed length $\ell=\theta_{B}-\theta_{A}$. To derive the PDF of $\delta \mathcal{N}_{m}$ in the limit $N \gg 1$ we will show that for scales larger than $1 / N$ the process $\delta \mathcal{N}_{\theta_{A}}(\theta)$ is very close to a special version of 

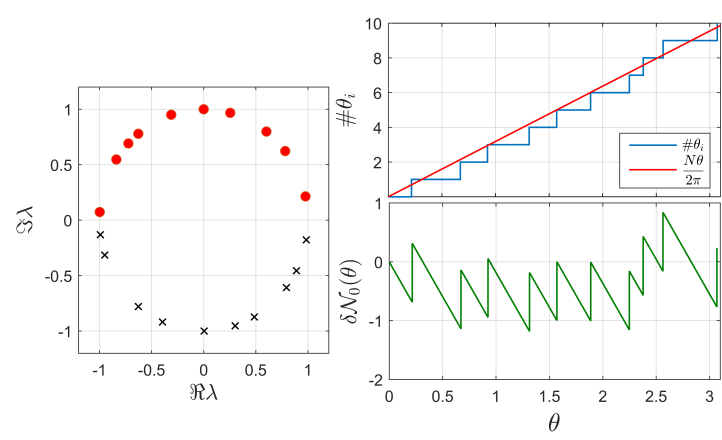

Figure 1: Constructing an instance of $\delta N_{0}(\theta)$ for $\theta \in[0, \pi]$ for $\beta=2$ and $N=20$. Left: eigenvalues $\lambda=e^{i \theta_{i}}$. Right: counting staircase (top), with mean subtracted (bottom).

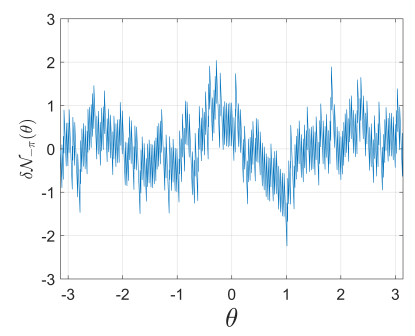

Figure 2: A single realization of $\delta N_{-\pi}(\theta)$ for the full circle $\theta \in[-\pi, \pi]$ for $\beta=2$ and $N=200$.

$1 D$ log-correlated Gaussian field, the so called fractional Brownian Motion with Hurst index $H=0$, denoted as $\mathrm{fBm} 0$, defined in [18] and whose extrema where investigated recently [19, 20]. However it turns out that the relation to $\mathrm{fBm} 0$ alone is insufficient to fully determine the statistics of $\delta \mathcal{N}_{m}$. Namely, we will demonstrate that although the process $\delta \mathcal{N}_{\theta_{A}}(\theta)$ for large $N \gg 1$ is very close to the $\mathrm{fBm} 0$ at different points, the non-Gaussian features which characterize its single-point statistics show up in a non-trivial way in the PDF of its maximum $\delta \mathcal{N}_{m}$. These single-point features are inherited from the discrete nature of the number of fermions/eigenvalues as exemplified e.g. in fermion counting statistics [2].

We now describe our main findings by first assuming that the Dyson parameter is rational and can be represented as $\beta / 2=s / r$ where $s$ and $r$ are mutually prime, and relaxing this assumption later on. We find that, for any fixed interval, the mean value of the maximum $\delta \mathcal{N}_{m}$ defined in (3) exhibits, for $N \rightarrow \infty$, the universal behavior of the log-correlated fields [21 24] :

$$
2 \pi \sqrt{\frac{\beta}{2}} \mathbb{E}\left(\delta \mathcal{N}_{m}\right) \simeq 2 \log N-\frac{3}{2} \log \log N+c_{\ell}^{(\beta)}
$$

where $c_{\ell}^{(\beta)}=O(1)$ is an unknown $\ell$-dependent constant. The variance for the maximum $\delta \mathcal{N}_{m}$ exhibits to the lead- ing order the extensive universal logarithmic growth typical for pinned log-correlated fields [19], on top of which we can evaluate the corrections of the order of unity:

$$
\mathbb{E}^{c}\left(\delta \mathcal{N}_{m}^{2}\right) \simeq \frac{2}{\beta(2 \pi)^{2}}\left(2 \log N+\tilde{C}_{2}^{(\beta)}+C_{2}(\ell)\right)
$$

Finally, the higher cumulants converge to a finite limit as $N \rightarrow \infty$ :

$$
\mathbb{E}^{c}\left(\delta \mathcal{N}_{m}^{k}\right) \simeq \frac{2^{k / 2}}{\beta^{k / 2}(2 \pi)^{k}}\left(\tilde{C}_{k}^{(\beta)}+C_{k}(\ell)\right)
$$

where the constants $C_{k}(\ell)=O(1)$ depend on the length $\ell$ of the interval and will be given below in two limiting cases. The $\ell$-independent constants $\tilde{C}_{k}^{(\beta)}$ for $k \geq 2$ are given by

$$
\tilde{C}_{k}^{(\beta)}=\left.\frac{d^{k}}{d t^{k}}\right|_{t=0} \log \left(A_{\beta}(t) A_{\beta}(-t)\right)
$$

where

$$
A_{\beta}(t)=r^{-t^{2} / 2} \prod_{\nu=0}^{r-1} \prod_{p=0}^{s-1} \frac{G\left(1-\frac{p}{s}+\frac{\nu+i t \sqrt{\frac{2}{\beta}}}{r}\right)}{G\left(1-\frac{p}{s}+\frac{\nu}{r}\right)}
$$

Here $G(z)$ denotes the standard Barnes function satisfying $G(z+1)=\Gamma(z) G(z)$, with $G(1)=1$. Note that all the odd coefficients $\tilde{C}_{2 k+1}^{(\beta)}$ vanish. Specifying for $\beta=2$, one has $A_{2}(t)=G(1+i t)$, leading to $\tilde{C}_{2}^{(2)}=2\left(1+\gamma_{E}\right)$ and $\tilde{C}_{4}^{(2)}=-12 \zeta(3)$. Notably, using $(7),(8)$, we were able to obtain a formula for the $\tilde{C}_{k}^{(\beta)}$ as single infinite series [27], which shows that they are smooth as a function of the Dyson parameter $\beta$, thus relaxing the assumption of rationality. As discussed below, the factors $A_{\beta}(t)$, hence $\tilde{C}_{k}^{(\beta)}$, are intimately but non-trivially related to the cumulants of the number of fermions (free for $\beta=2$ and with Sutherland-type interaction for $\beta \neq 2)$ in a mesoscopic interval of the circle.

By contrast the factors $C_{k}(\ell)$ are $\beta$-independent and originate from the problem of the maximum of a $\mathrm{fBm} 0$ on the interval $\left[\theta_{A}, \theta_{B}\right]$. For the $\ell$-dependent constants we obtain explicit formula in two cases:

(i) maximum over the full circle $\ell=2 \pi$. In that case $\left.\left.\left[\theta_{A}, \theta_{B}\right]=\right]-\pi, \pi\right]$ and we find for any $k \geq 2$

$$
C_{k}(2 \pi)=\left.(-1)^{k} \frac{d^{k}}{d t^{k}}\right|_{t=0} \log \left[\frac{\Gamma(1+t)^{2} G(2-2 t)}{G(2-t)^{3} G(2+t)}\right]
$$

which is related to the $\mathrm{fBm} 0$ bridge on $]-\pi, \pi]$ studied in 19

(ii) maximum over a mesoscopic interval $\frac{1}{N} \ll \ell \ll 1$.

For $k \geq 2$ we obtain in this regime

$$
\begin{aligned}
& C_{k}(\ell) \simeq 2 \log \ell \delta_{k, 2} \\
& +\left.(-1)^{k} \frac{d^{k}}{d t^{k}}\right|_{t=0}\left[\frac{2 \Gamma(1+t)^{2} G(2-2 t)}{G(2+t)^{2} G(2-t) G(4-t)}\right]
\end{aligned}
$$

This result is related to the $\mathrm{fBm} 0$ on an interval, with one pinned and one free end, studied in [19]. Note that the variance depends logarithmically on $\ell$ at small $\ell$, whereas 
higher cumulants have limits as $\ell \rightarrow 0$. Note that $l \rightarrow$ 0 limit is expected to provide the $L \gg 1$ asymptotic for statistics of the maximum of $\mathcal{N}_{\theta_{A}}(\theta)$ in intervals of the order $2 \pi L / N$, comparable with the mean eigenvalue spacing. The universal statistics of $\mathrm{CUE}_{\beta}$ eigenvalues at such local scales is described by the so called sine$\beta$ process [25] and the associated counting function has been studied in [26].

Finally, addressing the question of the location of the maximum in (3), $\theta_{m} \in\left[\theta_{A}, \theta_{B}\right]$, let us define $y_{m}=$ $\left(\theta_{m}-\theta_{A}\right) / \ell$. For the mesoscopic interval, we predict the PDF of $y_{m}$ to be symmetric around $\frac{1}{2}$, with $\mathbb{E}\left(y_{m}^{2}\right)=\frac{17}{50}$ and $\mathbb{E}\left(y_{m}^{4}\right)=\frac{311}{1470}$, thus deviating from the uniform distribution. For the full circle we find a uniform distribution for $\theta_{m}$ [28]. However, joint moments for the position and value of the maximum show the effect of pinning at $\theta=\theta_{A}$ (see details in [27]).

To elucidate the relation to $\mathrm{fBm} 0$, let us recall that the process $\delta N_{\theta_{A}}(\theta)$ is exactly given by the difference [27.

$$
\delta \mathcal{N}_{\theta_{A}}(\theta)=\frac{1}{\pi} \operatorname{Im} \log \xi_{N}(\theta)-\frac{1}{\pi} \operatorname{Im} \log \xi_{N}\left(\theta_{A}\right)
$$

where $\xi_{N}(\theta)=\operatorname{det}\left(1-e^{-i \theta} U\right)$ is the characteristic polynomial (CP). As shown in 29] for $\beta=2$ (see [30] for general $\beta>0$ ) the joint probability density of $\operatorname{Im} \log \xi_{N}(\theta)$ at two distinct points $\theta_{1} \neq \theta_{2}$ converges as $N \rightarrow+\infty$ to that of a Gaussian process $W_{\beta}(\theta)$ of zero mean and covariance

$$
\mathbb{E}\left(W_{\beta}\left(\theta_{1}\right) W_{\beta}\left(\theta_{2}\right)\right)=-\frac{1}{2 \beta} \log \left[4 \sin ^{2}\left(\frac{\theta_{1}-\theta_{2}}{2}\right)\right]
$$

a particular instance of the 1D log-correlated Gaussian field. Since 12 implies that $\delta \mathcal{N}_{\theta_{A}}\left(\theta=\theta_{A}\right)=0$ in any realization, the relevant object is the pinned log-correlated process closely related to $\mathrm{fBm} 0$. The log-correlated fields being highly singular always require a regularization to study their value distribution. The imaginary parts of the $\log \xi_{N}(\theta)$ for $N \gg 1$ provides such a natural regularization [30, 52 [54], being asymptotically a random process $W$ which shares the covariance 13 but with a finite variance $\mathbb{E}\left(W(\theta)^{2}\right)=\beta^{-1} \log N+O(1)$. Via 12 this provides the well-known asymptotic of the eigenvalues/fermions number variance: $\mathbb{E}\left(\delta \mathcal{N}^{2}(\theta)\right) \simeq$ $\frac{2}{\beta \pi^{2}} \log N$. We shall see however 27] that naively replacing the difference $\delta \mathcal{N}_{\theta_{A}}(\theta)$ with its Gaussian approximation $\frac{1}{\pi}\left[W_{\beta}(\theta)-W_{\beta}\left(\theta_{A}\right)\right]$ (related to the bosonization of the fermionic problem) is not sufficient for characterizing the maximum of the process.

Gaussian fields characterized by a logarithmic covariance appear in chaos and turbulence [31, branching random walks and polymers on trees [21, 22, multifractal disordered systems 32, 33, two-dimensional gravity [34, 35]. Early works on their extrema revealed a connection to a remarkable freezing transition [21, 22, 32. Through exact solutions, it led to predictions for the PDF of the maximum value of a log-correlated field on the cir- cle and on the interval 36, 37, involving the freezing duality conjecture (FDC) (see [20] for an extensive discussion). This led to further results in theoretical and mathematical physics [23, 38, 42] and probability [24, 43, 51]. A log-correlated context of random $\mathrm{CP}$ attracting a lot of attention [30, 45, 47, 55, 62], none of these studies yet addressed the eigenvalue/zeros counting function in the intervals $\ell=O(1)$.

To study the maximum of the random field $\delta \mathcal{N}(\theta)$ we follow [20, 36, 37, 55, 56] and introduce a statistical mechanics problem of partition sum:

$$
Z_{b}=\frac{N}{2 \pi} \int_{\theta_{A}}^{\theta_{B}} d \phi e^{2 \pi b \sqrt{\beta / 2} \delta \mathcal{N}_{\theta_{A}}(\phi)},
$$

The "inverse temperature" is equal to $-2 \pi b \sqrt{\beta / 2}$, and we choose $b>0$ since we are studying here the maximum retrieved from the free energy $\mathcal{F}$ for $b \rightarrow+\infty$ as

$$
\delta \mathcal{N}_{m}=\lim _{b \rightarrow+\infty} \mathcal{F} \quad, \quad \mathcal{F}=\frac{1}{2 \pi b \sqrt{\beta / 2}} \log Z_{b}
$$

To study the statistics of the associated free energy we start with considering the integer moments of $Z_{b}$ given by

$$
\begin{aligned}
& \mathbb{E}\left[Z_{b}^{n}\right]=\left(\frac{N}{2 \pi}\right)^{n} \int_{\theta_{A}}^{\theta_{B}} e^{-b \sqrt{\beta / 2} \sum_{a=1}^{n} N\left(\phi_{a}-\theta_{A}\right)} \prod_{a=1}^{n} d \phi_{a} \\
& \times \mathbb{E}\left[\prod_{j=1}^{N} e^{2 \pi b \sqrt{\beta / 2} \sum_{a=1}^{n}\left(\chi\left(\phi_{a}-\theta_{j}\right)-\chi\left(\theta_{A}-\theta_{j}\right)\right)}\right]
\end{aligned}
$$

The expectation value in 16 over the $\operatorname{CUE}_{\beta}(N)$ computed using (1) has the form $\mathbb{E}\left[\prod_{j=1}^{N} g\left(\theta_{j}\right)\right]$ where we defined

$$
\log g(\theta)=2 \pi b \sqrt{\beta / 2} \sum_{a=1}^{n}\left(\chi\left(\phi_{a}-\theta\right)-\chi\left(\theta_{A}-\theta\right)\right)
$$

This can be further rewritten for any $\left.\left.\phi_{a}, \theta, \theta_{A} \in\right]-\pi, \pi\right]$ with $\phi_{a}>\theta_{A}$ as

$$
\begin{aligned}
& \log g(\theta)=b \sqrt{\beta / 2}\left[\sum_{a=1}^{n} \phi_{a}-n \theta_{A}\right. \\
& \left.+n \arg e^{i\left(\theta_{A}-\theta+\pi\right)}-\sum_{a=1}^{n} \arg e^{i\left(\phi_{a}-\theta+\pi\right)}\right]
\end{aligned}
$$

where we define the arg function as

$$
\arg e^{i \phi}=\left\{\begin{array}{l}
\phi \quad-\pi<\phi \leq \pi \\
\phi-2 \pi \quad \pi<\phi \leq 3 \pi
\end{array}\right.
$$

For $\beta=2, \mathbb{E}\left[\prod_{j=1}^{N} g\left(\theta_{j}\right)\right]=\operatorname{det}_{1 \leq j, k \leq N}\left[g_{j-k}\right]$ is a Toeplitz determinant, where $g_{p}=\int_{-\pi}^{\pi} \frac{d \theta}{2 \pi} e^{-i p \theta} g(\theta)$ is the associated symbol, and $g(\theta)$ according to (18)-(19) has jump singularities. The corresponding asymptotics as $N \rightarrow \infty$ is given by the famous Fisher-Hartwig (FH) formula 64 proved rigorously in 65. For a general rational $\beta$ extension of $\mathrm{FH}$ formula has been conjectured in 63. Specifying the expressions in 63] to our case 
gives for $N \rightarrow+\infty$ and $n b^{2}<1$

$$
\begin{aligned}
& \mathbb{E}\left[Z_{b}^{n}\right] \simeq\left(\frac{N}{2 \pi}\right)^{n} N^{b^{2}\left(n+n^{2}\right)}\left|A_{\beta}(b)\right|^{2 n}\left|A_{\beta}(b n)\right|^{2} \\
& \times \int_{\theta_{A}}^{\theta_{B}} \prod_{1 \leq a<c \leq n}\left|1-e^{i\left(\phi_{a}-\phi_{c}\right)}\right|^{-2 b^{2}} \\
& \times \prod_{1 \leq a \leq n}\left|1-e^{i\left(\phi_{a}-\theta_{A}\right)}\right|^{2 n b^{2}} \prod_{a=1}^{n} d \phi_{a}
\end{aligned}
$$

where the function $A_{\beta}(b)$ is defined in (8). Had we used instead an approximation replacing the difference $\delta \mathcal{N}_{\theta_{A}}(\theta)$ in the large $-N$ limit with the logarithmically correlated Gaussian process $W_{\beta}(\theta)$ defined via $(12)$ - $(13)$, we would reproduce the Coulomb gas factors in (20) but miss the factors $A_{\beta}(b)$, see [27]. Hence, this product encapsulates the residual non-Gaussianity of the process.

Let us first discuss the simplest case $n=1$ when 20 . can be interpreted, via (14), as giving

$$
\mathbb{E}\left(e^{2 \pi b \delta \mathcal{N}_{\theta_{A}}(\theta)}\right) \simeq N^{2 b^{2}}\left|A_{\beta}(b)\right|^{4}\left(4 \sin ^{2} \frac{\theta-\theta_{A}}{2}\right)^{b^{2}}
$$

This formula can be interpreted as the generating function for the full counting statistics for the number of Sutherland-model fermions in an interval of size $\theta-\theta_{A}$ which seems not to be addressed in the literature apart from the free-fermion case $\beta=2[2,66$, and $\beta=4$ 67].

Further progress is possible in the two cases when the Coulomb integrals in 20 can be explicitly calculated.

(i) Full circle $\theta_{A}=-\pi, \theta_{B}=\pi$. In that case the Coulomb integral is known as the Morris integral 68] leading to

$$
\begin{aligned}
\mathbb{E}\left[Z_{b}^{n}\right] & \simeq\left(\frac{N}{2 \pi}\right)^{n} N^{b^{2}\left(n+n^{2}\right)}\left|A_{\beta}(b)\right|^{2 n}\left|A_{\beta}(b n)\right|^{2} \\
& \times \mathbf{M}(n, a=-n b, b)
\end{aligned}
$$

where $\mathbf{M}(n, a, b)$ is defined Eq (14) in [19]. This result is valid in the high temperature phase with $n b^{2}<1$. From this expression for integer moments there is a well defined procedure to obtain the double sided Laplace transform (DSLT) of the free energy first in the high temperature phase $b<1$ via an analytic continuation. Defining $t=$ $-b n$ we obtain

$$
\begin{aligned}
& \mathbb{E}\left(e^{-2 \pi \sqrt{\frac{\beta}{2}}\left(\mathcal{F}-\mathcal{F}_{1}\right) t}\right) \simeq N^{-t Q+t^{2}} A_{\beta}(t) A_{\beta}(-t) \\
& \times \Gamma(1+t b) \frac{G_{b}(Q-2 t) G_{b}(Q)^{3}}{G_{b}(Q-t)^{3} G_{b}(Q+t)}
\end{aligned}
$$

where $\mathcal{F}_{1}$ is a constant [69 and $Q=b+\frac{1}{b}$ and $G_{b}(x)$ is the generalized Barnes function, see Eq. (44) in [37] and [70. We note that if we multiply both sides of the equation by $\Gamma\left(1+\frac{t}{b}\right)$, the right hand side is invariant by duality $b \rightarrow$ $1 / b$, since formally $G_{b}(z)=G_{1 / b}(z)$. According to the FDC [20, 37] we obtain the DSLT in the low temperature phase $b>1$. The result can be written as

$$
\mathbb{E}\left(e^{-2 \pi \sqrt{\frac{\beta}{2}} \mathcal{F} t}\right) \Gamma\left(1+\frac{t}{b}\right)=\mathbb{E}\left(e^{-2 \pi \sqrt{\frac{\beta}{2}} \delta \mathcal{N}_{m} t}\right)
$$

where the r.h.s. is our main result, i.e. the DSLT of the PDF of $\delta \mathcal{N}_{m}$ for the full circle [71]

$$
\begin{aligned}
\mathbb{E}\left(e^{-2 \pi \sqrt{\frac{\beta}{2}} \delta \mathcal{N}_{m} t}\right) \simeq & N^{-2 t+t^{2}} e^{c t} A_{\beta}(t) A_{\beta}(-t) \\
& \times \frac{\Gamma(1+t)^{2} G(2-2 t)}{G(2-t)^{3} G(2+t)}
\end{aligned}
$$

which, according to 15 , is the $b \rightarrow+\infty$ limit of the l.h.s of (24). Here $c=\frac{3}{2} \log \log (N)+c^{\prime}$ and $c^{\prime}$ is a constant that we cannot determine by this method. Expansion of Eq. 25) around $t=0$ leads to the large $N$ asymptotics (4)-(6) for the cumulants, together with the predicted values for the coefficients $\tilde{C}_{k}^{(\beta)}$ in $(7)$ and $C_{k}(2 \pi)$ in $(9)$. The $C_{k}(2 \pi)$ equal, up to a factor $(-1)^{k}$, the cumulants $C_{k}$ given in [19] for the $\mathrm{fBm} 0$ bridge, checked against numerics there for $k=2,3,4$. These coefficients are studied in more details in [27].

(ii) Mesoscopic interval. A similar calculation gives the maximum over a mesoscopic interval $\frac{1}{N} \ll \ell \ll 2 \pi$. Relegating the details to [27] we simply quote our second main result, the DSLT of the PDF of $\delta \mathcal{N}_{m}$ for the small interval limit of small $\ell \ll 1$ :

$$
\begin{aligned}
& \mathbb{E}\left(e^{-2 \pi \sqrt{\frac{\beta}{2}} \delta \mathcal{N}_{m} t}\right) \simeq(N \ell)^{-2 t+t^{2}} e^{c t} A_{\beta}(t) A_{\beta}(-t) \\
& \Gamma(1+t)^{2} \frac{2 G(2-2 t)}{G(2+t)^{2} G(2-t) G(4-t)}
\end{aligned}
$$

where $c=\frac{3}{2} \log \log N+c^{\prime \prime}$. Expansion around $t=0$ leads to the same coefficients $\tilde{C}_{k}^{(\beta)}$ which are thus independent of $\ell$ (as can be seen already from (16)) and to the result for $C_{k}(\ell)$ in $10 p$, again related to the ones for the $\mathrm{fBm} 0$ on an interval given and numerically checked in [19. The structure of the above DSLT's in the complex plane for $t$ is discussed in [27].

In conclusion, we obtained the cumulants of the maximum of the deviation of the counting function from its mean on an interval, for eigenvalues of random unitary matrices and for free and interacting fermions on the circle. They inherit features both from the $\mathrm{fBm} 0$ log-correlated field and from the fermionic full counting statistics. Finally, our result for the distribution of $\delta \mathcal{N}_{m}$ provides a first step to study the Kolmogorov-Smirnov statistics for the counting staircases, which would further require the joint PDF of the maximum and minimum (usually non-trivially correlated [73]).

The results for the mesoscopic interval are expected to be universal for a broader class of random matrix ensembles, as well as for fermions on a lattice in the dilute limit [72. Finally, it is natural to conjecture that for $\beta=2$ universality extends to describing the statistics of the counting staircases for the nontrivial zeroes $t_{n}$ of the Riemann zeta-function $\zeta(1 / 2+i t)$ in mesoscopic intervals of the critical line $t \in \mathbb{R}$. Such zeroes are known to be 
extremely faithful to the random matrix statistics when analyzed in appropriate scales [74] underlying a fruitful line of applications of associated CP to understand ensuing features of $\zeta(1 / 2+i t)[29,30,75+78]$.

Acknowledgments: We thank X. Cao, J.P. Keating and G. Lambert for insightful comments on the early version of this paper, and S. B. Fedeli for his kind assistance with preparing figures. YVF thanks the Philippe Meyer Institute for Theoretical Physics at ENS in Paris. PLD acknowledges support from ANR grant ANR-17-CE300027-01 RaMa-TraF.

[1] L. S. Levitov and G. B. Lesovik. Charge distribution in quantum shot noise. JETP Lett. 58, No.3, 230-235 (1993) [Pis'ma v ZhETF 58, 225-230 (1993)].

[2] A. G. Abanov, D. A. Ivanov, Y. Qian. Quantum fluctuations of one-dimensional free fermions and FisherHartwig formula for Toeplitz determinants. J. Phys. A: Math. Theor. 44485001 (2011) arXiv:1108.1355.

[3] D. A. Ivanov and A. G. Abanov. Characterizing correlations with full counting statistics: Classical Ising and quantum XY spin chains. Phys. Rev. E 87, 022114 (2013).

[4] A. Bastianello, L. Piroli, and P. Calabrese. Exact Local Correlations and Full Counting Statistics for Arbitrary States of the One-Dimensional Interacting Bose Gas. Phys. Rev. Lett. 120, 190601 (2018).

[5] I. V. Protopopov, D. B. Gutman, and A. D. Mirlin. Luttinger liquids with multiple Fermi edges: Generalized Fisher-Hartwig conjecture and numerical analysis of Toeplitz determinants. Lith. J. Phys. 52, No. 2, pp. 165179 (2012) arXiv:1203.6418.

[6] V. Eisler. Universality in the full counting statistics of trapped fermions. Phys. Rev. Lett. 111, 080402 (2013) arXiv:1304.1413.

[7] R. Marino, S. N. Majumdar, G. Schehr, P. Vivo. Phase transitions and edge scaling of number variance in Gaussian random matrices. Phys. Rev. Lett. 112, 254101 (2014) arXiv:1404.0575.

[8] D. S. Dean, P. Le Doussal, S. N. Majumdar, G. Schehr. Non-interacting fermions at finite temperature in a ddimensional trap: universal correlations. Phys. Rev. A 94, 063622 (2016).

[9] O. Gamayun, O. Lychkovskiy and J.S. Caux. Fredholm determinants, full counting statistics and Loschmidt echo for domain wall profiles in one-dimensional free fermionic chains. arXiv:1911.01926.

[10] J.P. Keating, F. Mezzadri. Entanglement in Quantum Spin Chains, Symmetry Classes of Random Matrices, and Conformal Field Theory. Phys.Rev.Lett. 94, 050501 (2005).

[11] P. Calabrese, P. Le Doussal, S.N. Majumdar. Random matrices and entanglement entropy of trapped Fermi gases. Phys. Rev. A 91, 012303 (2015) arXiv:1411.4421.

[12] V. Eisler and Z. Racz. Full Counting Statistics in a Propagating Quantum Front and Random Matrix Spectra. Phys. Rev. Lett. 110, 060602 (2013).

[13] F. J. Dyson. Statistical Theory of the Energy Levels of Complex Systems I. J. Math. Phys. $3140-155$ (1962).
[14] R. Killip and I. Nenciu. Matrix Models for Circular Ensembles. Int. Math. Res. Notices 2004 No.50, 2665 2701 (2004).

[15] B. Sutherland. Exact Results for a Quantum Many-Body problem in One Dimension Phys. Rev. A 4, 2019-2021 (1971).

[16] Z. Bao, Y. He. On Cramer-von Mises statistic for the spectral distribution of random matrices. arXiv:1911.04151.

[17] T. Claeys, B. Fahs, G. Lambert, C. Webb. How much can the eigenvalues of a random Hermitian matrix fluctuate? arXiv:1906.01561

[18] Y. V. Fyodorov, B. A. Khoruzhenko, and N. J. Simm. Fractional Brownian motion with Hurst index $H=0$ and the Gaussian Unitary Ensemble. Ann. Probab. 44 2980-3031 (2016).

[19] X. Cao, Y. V. Fyodorov, and P. Le Doussal. Logcorrelated random-energy models with extensive freeenergy fluctuations: Pathologies caused by rare events as signatures of phase transitions, Phys. Rev. E 97, 022117 (2018) arXiv:1712.06023.

[20] Y.V. Fyodorov and P. Le Doussal. Moments of the position of the maximum for GUE characteristic polynomials and for log-correlated Gaussian processes. J. Stat. Phys. 164 190-240 (2016).

[21] B. Derrida, H. Spohn. Polymers on disordered trees, spin glasses, and travelling waves. J Stat Phys 51, 817-840 (1988).

[22] D. Carpentier and P. Le Doussal. Glass transition of a particle in a random potential, front selection in nonlinear renormalization group, and entropic phenomena in Liouville and sinh-Gordon models. Phys. Rev. E 63 026110 (2001).

[23] YV Fyodorov, P Le Doussal, A Rosso. Counting function fluctuations and extreme value threshold in multifractal patterns: the case study of an ideal $1 /$ f noise. J. Stat. Phys. 149 (5), 898-920 (2012).

[24] J. Ding, R. Roy, and O. Zeitouni. Convergence of the centered maximum of log-correlated Gaussian fields. Ann. Probab. 45, no. 6A, 3886-3928 (2017).

[25] B. Valko and B. Virag. Continuum limits of random matrices and the Brownian carousel. Inventiones Math., 177:463-508, 2009.

[26] D. Holcomb, E. Paquette. The maximum deviation of the Sine $\beta$ counting process. Electron. Commun. Probab. 23, no. $58,1-13$ (2018).

Note that the leading term in our equation (4) should be compared to the maximum of the "one-sided" process discussed in Prop. 1.5 of the above paper.

[27] see Supplementary material.

[28] To understand this, consider a discrete pinned field on the circle constructed as $V_{i}=U_{i}-U_{1}, i=1, \ldots M$ where $U_{i}$ is a standard centered log-correlated field. Clearly the position of the maximum for $U_{i}$, hence of the one for $V_{i}$, is uniformly distributed on the circle.

[29] C.P. Hughes, J.P. Keating and N O'Connell. On the characteristic polynomial of a random unitary matrix. Commun. Math. Phys. 220 429-451 (2001).

[30] R. Chhaibi, T. Madaule, and J. Najnudel. On the maximum of the $\mathrm{C} \beta \mathrm{E}$ field. Duke Math. J. 167, Number 12, 2243-2345 (2018).

[31] J.-P. Kahane. Sur le chaos multiplicatif. Ann. Sci. Math. Quebec. 9(2):105-150 (1985).

[32] C. Chamon, C. Mudry, X-G. Wen. Localization in two 
dimensions, gaussian field theories, and multifractality. Phys. Rev. Lett. 77 4194-4198 (1996).

[33] Y.V. Fyodorov. Multifractality and freezing phenomena in random energy landscapes: An introduction. Physica A 389 4229-4254 (2010).

[34] R. Rhodes, V. Vincent. Lecture notes on Gaussian multiplicative chaos and Liouville Quantum Gravity. in: Stochastic Processes and Random Matrices: Lecture Notes of Les Houches Summer School: July 2015. Session CIV. Ed. by G. Schehr et al. (Oxford University Press, 2017).

[35] A. Kupiainen, R. Rhodes, V. Vargas. Integrability of Liouville theory: proof of the DOZZ Formula. Ann. of Math. 191, No. 1, 81-166 (2020).

[36] Y.V. Fyodorov and J.-P. Bouchaud. Freezing and extreme-value statistics in a random energy model with logarithmically correlated potential. J. Phys. A: Math. Theor. 41372001 (2008).

[37] Y.V. Fyodorov, P. Le Doussal, and A. Rosso. Statistical mechanics of logarithmic REM: duality, freezing and extreme value statistics of $1 / f$ noises generated by Gaussian free fields. J Stat Mech 2009, P10005 (2009).

[38] D. Ostrovsky. On Barnes beta distributions and applications to the maximum distribution of the 2D Gaussian Free Field. J. Stat. Phys. 164 (2016) 1292-1317 (2016).

[39] D. Ostrovsky. A Review of Conjectured Laws of Total Mass of Bacry-Muzy GMC Measures on the Interval and Circle and Their Applications. Rev. Math. Phys. 30, No. 10, 1830003 (2018).

[40] X. Cao, P. Le Doussal, A. Rosso, R. Santachiara. Operator Product Expansion in Liouville Field Theory and Seiberg type transitions in log-correlated Random Energy Models. Phys. Rev. E 97, 042111 (2018) arXiv:1801.09991.

[41] X. Cao, P. Le Doussal, A. Rosso, R. Santachiara. Liouville field theory and log-correlated Random Energy Models. Phys. Rev. Lett. 118, 090601 (2017)[it arXiv:1611.02193.

[42] X. Cao, Y. V. Fyodorov, P. Le Doussal, One step replica symmetry breaking and extreme order statistics of logarithmic REMs. SciPost Phys. 1, 011 (2016) arXiv:1610.02226.

[43] T. Madaule, R. Rhodes, V. Vargas. Glassy phase and freezing of log-correlated Gaussian potentials. Ann. Appl. Probab. 26 No. 2, 643-690 (2016)

[44] N. Berestycki.An elementary approach to Gaussian multiplicative chaos. Electron. Commun. Probab. 22, paper no. 27, 12 pp (2017)

[45] Y V Fyodorov and N J Simm. On the distribution of the maximum value of the characteristic polynomial of GUE random matrices. Nonlinearity 29 2837-2855 (2016)

[46] L.-P. Arguin, D. Belius, and P. Bourgade. Maximum of the characteristic polynomial of random unitary matrices. Comm. Math. Phys. 349, no. 2, 703-751 (2017)

[47] E. Paquette, O. Zeitouni. The Maximum of the CUE Field. Int. Math. Res. Notices, 2018 (16), 5028-5119 (2018)

[48] E. Subag, O. Zeitouni. Freezing and decorated Poisson point processes. Commun. Math. Phys. 337 (1), 55-92 (2015)

[49] G. Remy. The Fyodorov-Bouchaud formula and Liouville conformal field theory. Duke Math. J. 169, Number 1 , 177-211 (2020) arXiv:1710.06897.

[50] G. Remy, T. Zhu. The distribution of Gaussian multi- plicative chaos on the unit interval arXiv:1804.02942

[51] M. Biskup, O. Louidor. Full extremal process, cluster law and freezing for the two-dimensional discrete Gaussian Free Field. Adv. Math. 330, 589-687 (2018).

[52] C. Webb. The characteristic polynomial of a random unitary matrix and Gaussian multiplicative chaos - the $L^{2}$ phase. Electron. J. Probab. 20104 (2015).

[53] N. Berestycki, C. Webb, and M. D. Wong. Random Hermitian matrices and Gaussian multiplicative chaos. Probab. Theor. Rel. Fields 172, 103-189 (2018) [https://doi.org/10.1007/s00440-017-0806-9]

[54] G. Lambert. Mesoscopic central limit theorem for the circular beta-ensembles and applications. arXiv:1902.06611

[55] Y.V. Fyodorov, G.A. Hiary, and J.P. Keating. Freezing transitions, characteristic polynomials of random matrices, and the Riemann zeta-function. Phys. Rev. Lett. 108, 170601 (2012).

[56] Y.V. Fyodorov and J.P. Keating. Freezing transitions and extreme values: random-matrix theory, $\zeta\left(\frac{1}{2}+i t\right)$ and disordered landscapes. Phil. Trans. Roy. Soc. A 372, 20120503 (2014).

[57] L.-P. Arguin, D. Belius, and A. J. Harper. Maxima of a randomized Riemann zeta function, and branching random walks. Ann. Appl. Probab. 27, no. 1, 178-215 (2017).

[58] L.-P. Arguin, D. Belius, P. Bourgade, M.Radziwill and K. Soundararajan. Maximum of the Riemann zeta function on a short interval of the critical line. Commun. Pure. Appl. Math 72, Issue 3, 500-535 (2019)

[59] J. Najnudel. On the extreme values of the Riemann zeta function on random intervals of the critical line. Probab. Theory Relat. Fields 172, Issue 1-2, 387-452(2018) [ https://doi.org/10.1007/s00440-017-0812-y]

[60] L.-P. Arguin, F. Ouimet, M. Radziwill. Moments of the Riemann zeta function on short intervals of the critical line. arXiv:1901.04061

[61] A.J. Harper. On the partition function of the Riemann zeta function, and the Fyodorov-Hiary-Keating conjecture. arXiv:1906.05783

[62] R. Chhaibi, J. Najnudel. On the circle, $G M C^{\gamma}=$ $\lim C \beta E_{n}$ for $\gamma=\sqrt{2 / \beta},(\gamma \leq 1)$. arXiv:1904.00578

[63] P.J. Forrester and N.E. Frankel. Applications and generalizations of Fisher-Hartwig asymptotics. J. Math. Phys. 45, 2003-2028 (2004).

[64] M.E. Fisher and R. E. Hartwig. Toeplitz determinants: some applications, theorems, and conjectures. Advances in Chemical Physics: Stochastic processes in chemical physics (1969): 333-353 (1969)

[65] P. Deift, A. Its, I. Krasovsky. Asymptotics of Toeplitz, Hankel, and Toeplitz+Hankel determinants with FisherHartwig singularities Ann. of Math. 174, 1243-1299 (2011) arXiv:0905.0443.

[66] For $\beta=2$ and small $\theta-\theta_{A}$ our result 21 coincides with [2] in the low density limit $k_{F} \rightarrow 0$ (setting $2 L k_{F}=$ $N \ell$. Note that in 2] each factor $|G(1+i \gamma)|^{2}$ is meant as $G(1+i \gamma) G(1-i \gamma)$, valid more generally for $\gamma$ in the complex plane, limited however to a strip.

[67] J.-M. Stephan and F. Pollmann. Full counting statistics in the Haldane-Shastry chain. Phys. Rev. B 95, 035119 (2017).

[68] P. Forrester and S. Warnaar. The importance of the Selberg integral. Bull. Amer. Math. Soc. (N.S.) 45, 489-534 (2008) arXiv:0710.3981.

[69] We defined $\mathcal{F}_{1}=\frac{1}{\pi b \sqrt{2 \beta}} \log K_{\beta}(b)$ with $K_{\beta}(b)=$ 
$\left|A_{\beta}(b)\right|^{2} /\left(2 \pi \Gamma\left(1-b^{2}\right)\right)$ and similarly for $\tilde{\mathcal{F}}_{1}$ with $\tilde{K}_{\beta}(b)=K_{\beta}(b)(2 \pi)^{\beta-1}$

[70] in [19], Eq. (15), $\tilde{G}_{\beta}(z)=\beta^{\frac{z^{2}}{2}-\frac{z}{2} Q}(2 \pi)^{z\left(\frac{1}{2 \beta}-\frac{1}{2}\right)} G_{\beta}(z)$ was used, however one can check that for Eq. 23 it is immaterial (see also Section 13.2 in 20]).

[71] The DSLT for complex $t$ is discussed in [27.

[72] possibly beyond, up to a change in the log term of the second cumulant.

[73] X. Cao, P. Le Doussal. Joint min-max distribution and Edwards-Anderson's order parameter of the circular $1 / f$ noise model. EPL (Europhysics Letters) 114 (4), 40003 (2016)

[74] A. M. Odlyzko. On the distribution of spacings between zeros of the zeta function. Math. Comp., 48, 273-308 (1987).

[75] J.P. Keating and N.C. Snaith. Random matrix theory and $\zeta(1 / 2+i t)$ Commun. Math. Phys. 214 57-89 (2000).

[76] J.P. Keating. Random matrices and number theory: some recent themes. in: Stochastic Processes and Random Matrices: Lecture Notes of Les Houches Summer School: July 2015. Session CIV. Ed. by G. Schehr et al. (Oxford University Press, 2017).

[77] P. Bourgade. Mesoscopic fluctuations of the zeta zeros. Probab. Theory Rel. Fields 148, no. 3-4, 479-500 (2010).

[78] P. Bourgade and J. Kuan. Strong Szego asymptotics and zeros of the zeta-function. Comm. Pure Appl. Math. 67, no. 6, 1028-1044 (2014). 


\section{SUPPLEMENTARY MATERIAL}

\section{Statistics of extremes in eigenvalue-counting staircases}

Yan V. Fyodorov and P. Le Doussal

We provide some additional details for some of the calculations described in the manuscript of the Letter.

\section{CUMULANT AMPLitudes $\tilde{C}_{k}^{(\beta)}$ AS A FUNCTION OF $\beta$}

Let us recall the formula given in the text for the coefficients $\tilde{C}_{k}^{(\beta)}$ which enter in the cumulants of the PDF for $\delta \mathcal{N}_{m}$, namely, for $\beta=2 s / r$, with $s, r$ mutually prime and $k \geq 2$

$$
\begin{gathered}
\tilde{C}_{k}^{(\beta)}=\left.\frac{d^{k}}{d t^{k}}\right|_{t=0} \log \left(A_{\beta}(t) A_{\beta}(-t)\right) \\
A_{\beta}(t)=r^{-t^{2} / 2} \prod_{\nu=0}^{r-1} \prod_{p=0}^{s-1} \frac{G\left(1-\frac{p}{s}+\frac{\nu+i t \sqrt{\frac{2}{\beta}}}{r}\right)}{G\left(1-\frac{p}{s}+\frac{\nu}{r}\right)}
\end{gathered}
$$

To obtain more explicit expressions we use that for $k \geq 2$

$$
\left.\frac{d^{k}}{d y^{k}} \log G(x+y)\right|_{y=0}=\phi_{k}(x):=(k-1) \psi^{(k-2)}(x)+(x-1) \psi^{(k-1)}(x)-\delta_{k, 2}
$$

where $\psi^{(k)}(x)=\frac{d^{k+1}}{d x^{k+1}} \log \Gamma(x)$. Hence, for even $k=2 p$, defining $p=s-q$ we obtain

$$
\tilde{C}_{2 p}^{(\beta)}=(-1)^{p} \frac{2}{(r s)^{p}} \sum_{\nu=0}^{r-1} \sum_{q=1}^{s} \phi_{2 p}\left(\frac{\nu}{r}+\frac{q}{s}\right)-2 \log r \delta_{p, 1}
$$

and we recall that odd cumulants vanish.

Since any real $\beta$ can be reached by a sequence $\beta=2 s_{n} / r_{n}$ of arbitrary large $s_{n}, r_{n}$ we can obtain an alternative expression valid for any $\beta$ in terms of a convergent infinite series. We need to distinguish two cases:

Cumulants $C_{2 p}$ with $p \geq 2$. In that case we see that the large $s, r$ behavior in 30 is dominated by the divergence of $\phi_{k}(x)$ near $x=0$. We use that

$$
\phi_{2 p}(x)=-\frac{(2 p-1) !}{x^{2 p}}+O(1)
$$

One finds for $k=2 p$ with $p \geq 2$

$$
\tilde{C}_{2 p}^{(\beta)}=(-1)^{p+1} 2(2 p-1) ! \sum_{\nu=0}^{\infty} \sum_{q=1}^{\infty} \frac{1}{\left(\nu \sqrt{\frac{\beta}{2}}+q \sqrt{\frac{2}{\beta}}\right)^{2 p}}
$$

One of the sum can be carried out leading to two equivalent "dual" expressions

$$
\tilde{C}_{2 p}^{(\beta)}=(-2)^{1-p} \beta^{p} \sum_{\nu=0}^{\infty} \psi^{(2 p-1)}\left(1+\frac{\beta \nu}{2}\right)=(-2)^{p+1} \frac{1}{\beta^{p}} \sum_{q=1}^{\infty} \psi^{(2 p-1)}\left(\frac{2 q}{\beta}\right)
$$

where we have used that $\psi^{(2 p-1)}(1)=(2 p-1) ! \zeta(2 p)$. The above series are convergent for $p \geq 2$, since at large $x$ one has $\psi^{(2 p-1)}(x) \simeq \frac{(2 p-2) !}{z^{2 p-1}}$. Hence the result is analytic in $\beta>0$. This asymptotics can be used to obtain the large $\beta$ expansion

$$
\tilde{C}_{2 p}^{(\beta)}=(-2)^{1-p}(2 p-1) ! \zeta(2 p) \beta^{p}+(-1)^{p+1} 2^{p}(2 p-2) ! \zeta(2 p-1) \frac{1}{\beta^{p-1}}+O\left(\beta^{-p}\right)
$$

as well as the small $\beta$ expansion

$$
\tilde{C}_{2 p}^{(\beta)} \simeq(-1)^{p+1} 2^{2-p}(2 p-2) ! \zeta(2 p-1) \beta^{p-1} \quad, \quad \beta \ll 1
$$


As an example we give more explicitly the fourth cumulant

$$
\tilde{C}_{4}^{(\beta)}=-12 \sum_{\nu=0}^{\infty} \sum_{q=1}^{\infty} \frac{1}{\left(\nu \sqrt{\frac{\beta}{2}}+q \sqrt{\frac{2}{\beta}}\right)^{4}}=-\frac{8}{\beta^{2}} \sum_{q=1}^{\infty} \psi^{(3)}\left(\frac{2 q}{\beta}\right)=-\frac{1}{2} \beta^{2} \sum_{\nu=0}^{\infty} \psi^{(3)}\left(1+\frac{\beta \nu}{2}\right)
$$

One can then check that this formula, valid for any $\beta$, correctly reproduces for the cases $\beta=2 s / r$, with $s, r$ mutual primes, the same result as the original formula (30), for instance one finds

$$
\tilde{C}_{4}^{(\beta=2)}=-12 \zeta(3) \quad, \quad \tilde{C}_{4}^{(\beta=1)}=\frac{\pi^{4}}{4}-24 \zeta(3) \quad, \quad \tilde{C}_{4}^{(\beta=4)}=-24 \zeta(3)-\frac{\pi^{4}}{4}
$$

Let us also give more detailed asymptotics at large and small $\beta$

$$
\begin{aligned}
& \tilde{C}_{4}^{(\beta)}=-\frac{1}{30} \pi^{4} \beta^{2}-\frac{8 \zeta(3)}{\beta}+\frac{4 \pi^{4}}{15 \beta^{2}}-\frac{32 \zeta(5)}{\beta^{3}}+O\left(\beta^{-4}\right) \\
& =-2 \beta \zeta(3)-\frac{\pi^{4} \beta^{2}}{60}-\frac{\beta^{3} \zeta(5)}{2}+O\left(\beta^{5}\right)
\end{aligned}
$$

The fourth cumulant is plotted as a function of $\beta$ in the Figure 3 together with the large and small $\beta$ asymptotics which, as we see, are quite accurate.

Second cumulant $\tilde{C}_{2}^{(\beta)}$. The second cumulant reads, for $\beta=2 s / r$

$$
\tilde{C}_{2}^{(\beta)}=-\frac{2}{r s} \sum_{\nu=0}^{r-1} \sum_{q=1}^{s} \phi_{2}\left(\frac{\nu}{r}+\frac{q}{s}\right)-2 \log r
$$

To study the limit where both $r, s \rightarrow+\infty$ with a fixed (more precisely, converging) ratio $\beta=2 s / r$, it is useful to decompose $\phi_{2}(x)=-\frac{1}{x^{2}}+\tilde{\phi}_{2}(x)$, where $\tilde{\phi}_{2}(x)$ is regular at $x=0$, and to introduce $\sum_{\nu=0}^{r-1} \frac{1}{1+\nu}=H_{r} \simeq \log r+\gamma_{E}+$ $O(1 / r)$. Then one has in that limit

$$
-\frac{2}{r s} \sum_{\nu=0}^{r-1} \sum_{q=1}^{s} \tilde{\phi}_{2}\left(\frac{\nu}{r}+\frac{q}{s}\right) \rightarrow-2 \int_{0}^{1} d x \int_{0}^{1} d y \tilde{\phi}_{2}(x+y)=-2\left(\int_{0}^{1} d s s \tilde{\phi}_{2}(s)+\int_{1}^{2} d s(2-s) \tilde{\phi}_{2}(s)\right)=2 \log 2
$$

Hence need to evaluate the limit

$$
\begin{aligned}
& \tilde{C}_{2}^{(\beta)} \simeq 2 \log 2+2 \gamma_{E}+2 \sum_{\nu=0}^{r-1}\left[\sum_{q=1}^{s} \frac{\beta / 2}{\left(\nu \frac{\beta}{2}+q\right)^{2}}-\frac{1}{1+\nu}\right] \\
& =2 \log 2+2 \gamma_{E}+2 \sum_{\nu=0}^{r-1}\left[\frac{\beta}{2} \psi^{(1)}\left(1+\frac{\beta \nu}{2}\right)-\frac{\beta}{2} \psi^{(1)}\left(1+s+\frac{\beta \nu}{2}\right)-\frac{1}{1+\nu}\right]
\end{aligned}
$$

where we have used that $\sum_{q=1}^{s} \frac{1}{(q+a)^{2}}=\psi^{(1)}(1+a)-\psi^{(1)}(1+s+a)$. Now one can check that

$$
\lim _{r \rightarrow+\infty} \sum_{\nu=0}^{r-1} \frac{\beta}{2} \psi^{(1)}\left(1+\frac{\beta}{2} r+\frac{\beta \nu}{2}\right) \simeq \lim _{r \rightarrow+\infty} \sum_{p=r}^{2 r-1} \frac{1}{\frac{2}{\beta}+p}=\log 2
$$

where the second line is obtained writing $p=r+\nu$ and using $\psi^{(1)}(x) \sim 1 / x$ at large $x$, but the full equivalence has also been confirmed numerically. Hence we can take the large $r, s$ limit in 42 , the factors $\log 2$ cancel, and we finally obtain the second cumulant for any $\beta$ as the following convergent "dual" series

$$
\begin{aligned}
\tilde{C}_{2}^{(\beta)}=2 \gamma_{E}+2 \sum_{\nu=0}^{+\infty}\left[\sum_{q=1}^{+\infty} \frac{\beta / 2}{\left(\nu \frac{\beta}{2}+q\right)^{2}}-\frac{1}{1+\nu}\right] & =2 \gamma_{E}+2 \sum_{\nu=0}^{+\infty}\left[\frac{\beta}{2} \psi^{(1)}\left(1+\frac{\beta \nu}{2}\right)-\frac{1}{1+\nu}\right] \\
& =2 \gamma_{E}+2 \log (\beta / 2)+2 \sum_{q=1}^{\infty}\left(\frac{2}{\beta} \psi^{(1)}\left(\frac{2 q}{\beta}\right)-\frac{1}{q}\right)
\end{aligned}
$$

Note the non trivial term $2 \log (\beta / 2)$ in the last expression, arising from the replacement $-2 \log r=-2 \log s+2 \log (\beta / 2)$ in 40 . For $\beta=2$ one recovers $\tilde{C}_{2}^{(\beta=2)}=2+2 \gamma_{E}$. We also find either from 45, or from the original formula 40,

$$
\tilde{C}_{2}^{(\beta=1)}=2+2 \gamma_{E}-\frac{\pi^{2}}{4} \quad, \quad \tilde{C}_{2}^{(\beta=4)}=2+2 \gamma_{E}+\frac{\pi^{2}}{4}+\log (4)
$$


One obtains the series at large and small $\beta$

$$
\begin{aligned}
& \tilde{C}_{2}^{(\beta)}=\frac{\pi^{2} \beta}{6}+2 \gamma_{E}-\frac{\pi^{2}}{3 \beta}+\frac{4 \zeta(3)}{3 \beta^{2}}-\frac{16 \zeta(5)}{15 \beta^{4}}+O\left(\beta^{-5}\right) \\
& =2 \log (\beta / 2)+2 \gamma_{E}+\frac{\pi^{2} \beta}{12}+\frac{\beta^{2} \zeta(3)}{12}-\frac{\beta^{4} \zeta(5)}{240}+O\left(\beta^{5}\right)
\end{aligned}
$$

Note that the leading term agrees with 40 although that result assumed $p \geq 2$.

The second cumulant is plotted as a function of $\beta$ in the Figure 3 together with the large and small $\beta$ asymptotics which, as we see, are again quite accurate.

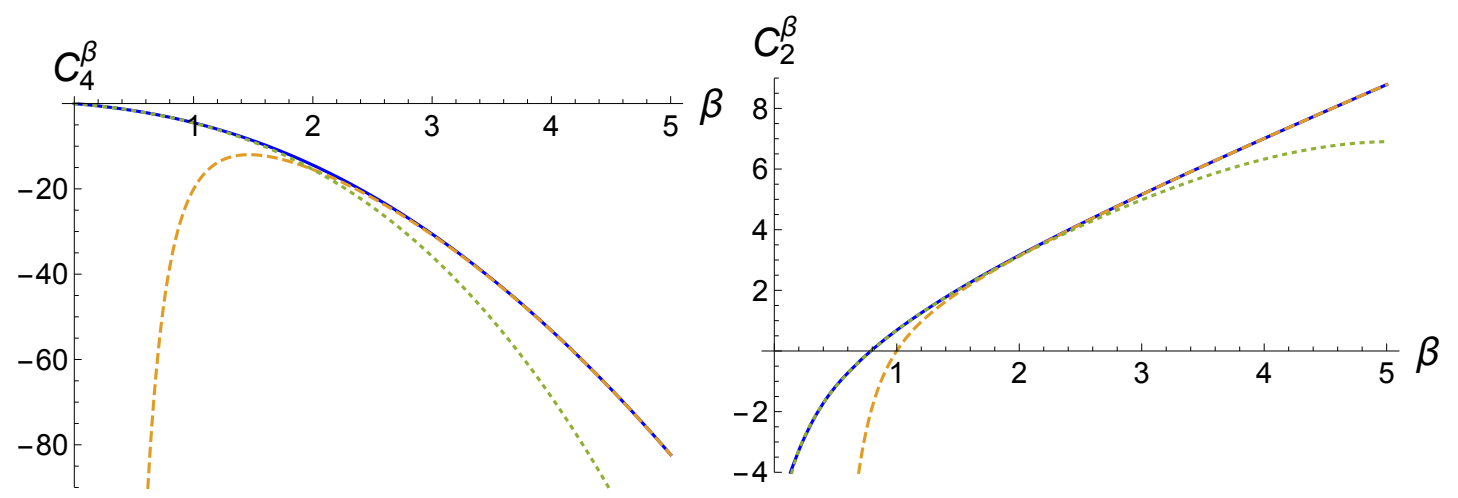

Figure 3: Left: fourth cumulant amplitude $\tilde{C}_{4}^{(\beta)}$ plotted (in blue) as a function of $\beta$ from $(36$ ). Dotted and dashed lines are small and large $\beta$ asymptotics, 38 respectively. Right: same for second cumulant amplitude $\tilde{C}_{2}^{(\beta)}$ (in blue) from 445 and 48 .

\section{CUMULANT AMPLITUDES $C_{k}(\ell)$}

Let us recall the result given in the text for the amplitudes $C_{k}(\ell)$ for $\ell=2 \pi$ and $\ell \ll 1$. For any $k \geq 2$

$$
\begin{aligned}
& C_{k}(2 \pi)=\left.(-1)^{k} \frac{d^{k}}{d t^{k}}\right|_{t=0} \log \left[\frac{\Gamma(1+t)^{2} G(2-2 t)}{G(2-t)^{3} G(2+t)}\right] \\
& C_{k}(\ell) \simeq 2 \log \ell \delta_{k, 2}+\left.(-1)^{k} \frac{d^{k}}{d t^{k}}\right|_{t=0}\left[\frac{2 \Gamma(1+t)^{2} G(2-2 t)}{G(2+t)^{2} G(2-t) G(4-t)}\right]
\end{aligned}
$$

We now use Eq. 29p, and we also use that $\psi^{(k)}(1)=(-1)^{k+1} k ! \zeta(k+1)$ and $\psi^{(k)}(2)=\psi^{(k)}(1)+(-1)^{k} k !$ and $\psi^{(k)}(4)=\psi^{(k)}(1)+(-1)^{k} k !\left(1+2^{-k-1}+3^{-k-1}\right)$. We obtain for the full circle

$$
\begin{aligned}
& C_{2}(2 \pi)=\frac{\pi^{2}}{3} \quad, \quad C_{3}(2 \pi)=2 \pi^{2}-8 \zeta(3) \quad, \quad C_{4}(2 \pi)=\frac{14}{15} \pi^{4}-72 \zeta(3) \\
& C_{k}(2 \pi)=\left(\left(1-(-2)^{k}+3(-1)^{k}\right) \zeta(k-1)+\left(1+(-2)^{k}-3(-1)^{k}\right) \zeta(k)\right) \Gamma(k) \quad, \quad k \geq 3
\end{aligned}
$$

and for the mesoscopic interval $\ell \ll 1$

$$
\begin{aligned}
& C_{2}(\ell)=2 \log \ell+\frac{9}{4}, \quad C_{3}(\ell)=-\frac{17}{4}+\frac{8 \pi^{2}}{3}-8 \zeta(3) \quad, \quad C_{4}(\ell)=\frac{99}{8}+\frac{4}{5} \pi^{4}-72 \zeta(3) \\
& C_{k}(\ell)=6^{-k}\left(\left(-(-12)^{k}+(-3)^{k} 2^{k+1}+2^{k+1} 3^{k}\right) \zeta(k-1)+(-6)^{k}\left(2^{k}-4\right) \zeta(k)+(-3)^{k}\left(2^{k+1}+1\right)\right) \Gamma(k), k \geq 3
\end{aligned}
$$

DISTRIBUTION OF THE MAXIMUM OVER A MESOSCOPIC INTERVAL $\frac{1}{N} \ll \ell \ll 2 \pi$

Here we sketch the derivation of our second main result, 26.

To this end we set $\phi_{a}=\theta_{A}+\ell x_{a}$, with $x_{a} \in[0,1]$, and recall $\ell=\theta_{B}-\theta_{A}$. Eq (20) gives

$$
\mathbb{E}\left[Z_{b}^{n}\right] \simeq\left(\frac{N \ell}{2 \pi}\right)^{n}(N \ell)^{b^{2}\left(n+n^{2}\right)}\left|A_{\beta}(b)\right|^{2 n}\left|A_{\beta}(b n)\right|^{2} \prod_{a=1}^{n} \int_{0}^{1} d x_{a} \prod_{1 \leq a<c \leq n}\left|x_{a}-x_{c}\right|^{-2 b^{2}} \prod_{1 \leq a \leq n} x_{a}^{2 n b^{2}}
$$


One recognizes now the Selberg integral [68] in the form which arises in the study of the fBm0 on an interval [19, 20]. Using the known expression for its analytical continuation (see Eq. (239) in [20]) and following the same steps as for the full circle presented in the text, we obtain the DSLT in the high temperature phase $b<1$ with $t=-n b$ as 69

$$
\mathbb{E}\left(e^{-2 \pi \sqrt{\frac{\beta}{2}}\left(\mathcal{F}-\tilde{\mathcal{F}}_{1}\right) t}\right) \simeq(N \ell)^{-t Q+t^{2}}\left|A_{\beta}(-t)\right|^{2} \Gamma(1+t b) \frac{G_{b}(Q)^{2} G_{b}(Q-2 t) G_{b}(2 Q)}{G_{b}(Q+t)^{2} G_{b}(Q-t) G_{b}(2 Q-t)}
$$

The duality invariance can be similarly checked and from the FDC we find that the DSLT in the low temperature phase $b>1$ is given again by (24) with our second main result, i.e. Eq. 26] of the main text.

\section{VERIFICATION OF RELATION EQ.(12)}

Our starting point is the characteristic polynomial defined in the text, which we rewrite

$$
\begin{aligned}
& \xi_{N}(\theta)=\prod_{j}\left(1-e^{i\left(\theta_{j}-\theta\right)}\right)=\prod_{j} e^{i\left(\theta_{j}-\theta-\pi\right) / 2} 2 \sin \frac{\theta_{j}-\theta}{2} \\
& =e^{-i \frac{N(\pi+\theta)}{2}+\frac{i}{2} \sum_{j=1}^{N} \theta_{j}} \prod_{j=1}^{N} \operatorname{sgn}\left[\sin \frac{\theta_{j}-\theta}{2}\right] \prod_{j=1}^{N} 2\left|\sin \frac{\theta_{j}-\theta}{2}\right|
\end{aligned}
$$

Further using

$$
\prod_{j=1}^{N} \operatorname{sgn}\left[\sin \frac{\theta_{j}-\theta}{2}\right]=\prod_{j=1}^{N} \operatorname{sgn}\left(\theta_{j}-\theta\right)=(-1)^{\#\left(\theta_{j}<\theta\right)}=e^{i \pi \#\left(\theta_{j}<\theta\right)}
$$

where $\#\left(\theta_{j}<\theta\right):=\sum_{j=1}^{N} \chi\left(\theta-\theta_{j}\right)$ is the number of $\theta_{j}$ not exceeding the value $\theta$, we see that

$$
\operatorname{Im} \log \xi_{N}(\theta)=-\frac{N}{2}(\pi+\theta)+\frac{1}{2} \sum_{j=1} \theta_{j}+\pi \#\left(\theta_{j}<\theta\right)
$$

implying via the definition 2

$$
\frac{1}{\pi} \operatorname{Im} \log \xi_{N}(\theta)-\frac{1}{\pi} \operatorname{Im} \log \xi_{N}\left(\theta_{A}\right)=-\frac{N}{2 \pi}\left(\theta-\theta_{A}\right)+\#\left(\theta_{j}<\theta\right)-\#\left(\theta_{j}<\theta_{A}\right):=\delta \mathcal{N}_{\theta_{A}}(\theta)
$$

exactly as claimed in 12 .

\section{DEFICIENCY OF THE LOG-CORRELATED GAUSSIAN APPROXIMATION FOR CHARACTERISING THE MAXIMUM OF $\delta \mathcal{N}_{\theta_{A}}(\theta)$.}

Let us demonstrate that naively replacing the difference $\delta \mathcal{N}_{\theta_{A}}(\theta)$ with its Gaussian approximation $\frac{1}{\pi}\left[W_{\beta}(\theta)-W_{\beta}\left(\theta_{A}\right)\right]$ is not sufficient for the purpose of characterizing the maximum of the process. For this end, we make the corresponding replacement in the expression (first line of 16 for the integer moments of $Z_{b}$, yielding

$$
\mathbb{E}\left[Z_{b}^{n}\right]=\left(\frac{N}{2 \pi}\right)^{n} \int_{\theta_{A}}^{\theta_{B}} \mathbb{E}\left[e^{2 b \sqrt{\beta / 2}\left(\sum_{a=1}^{n} W_{\beta}\left(\phi_{a}\right)-n W_{\beta}\left(\theta_{A}\right)\right)}\right] \prod_{a=1}^{n} d \phi_{a}
$$

where now the expectation is over the mean-zero Gaussian process $W_{\beta}(\theta)$ with the covariance given by $(13)$ and the variance $\mathbb{E}\left(W(\theta)^{2}\right)=\beta^{-1} \log N$. Due to Gaussian nature of the process the expectation is readily taken via the identity

$$
\mathbb{E}\left[e^{2 b \sqrt{\beta / 2}\left(\sum_{a=1}^{n} W_{\beta}\left(\phi_{a}\right)-n W_{\beta}\left(\theta_{A}\right)\right)}\right]=e^{\beta b^{2}\left[n(n+1) \mathbb{E}\left(W\left(\theta_{A}\right)^{2}\right)+2 \sum_{a<c}^{n} \mathbb{E}\left(W\left(\phi_{a}\right) W\left(\phi_{c}\right)\right)-2 n \sum_{a=1}^{n} \mathbb{E}\left(W\left(\phi_{a}\right) W\left(\theta_{A}\right)\right)\right]}
$$


Substituting here the value $\left[13\right.$ for the covariance and the associated variance and recalling that $4 \sin ^{2}\left(\frac{\theta_{1}-\theta_{2}}{2}\right)=$ $\left|e^{i \theta_{1}}-e^{i \theta_{2}}\right|^{2}$ we immediately arrive at the expression for the moments:

$$
\begin{aligned}
& \mathbb{E}\left[Z_{b}^{n}\right] \simeq\left(\frac{N}{2 \pi}\right)^{n} N^{b^{2} n(n+1)} \int_{\theta_{A}}^{\theta_{B}} \prod_{1 \leq a<c \leq n}\left|1-e^{i\left(\phi_{a}-\phi_{c}\right)}\right|^{-2 b^{2}} \\
& \times \prod_{1 \leq a \leq n}\left|1-e^{i\left(\phi_{a}-\theta_{A}\right)}\right|^{2 n b^{2}} \prod_{a=1}^{n} d \phi_{a}
\end{aligned}
$$

which misses exactly the factor $\left|A_{\beta}(b)\right|^{2 n}\left|A_{\beta}(b n)\right|^{2}$ when compared to the formula $[20)$. As those factors contribute to the cumulants for the maximum of the process, the Gaussian approximation is clearly insufficient for this purpose.

\section{DSLT IN THE COMPLEX PLANE}

The formulas (23), (25), (26), of the main text for the DSLT were obtained for real values of the parameter $t$. We expect them to extend to a domain around $t=0$ in the complex plane. For real $t$ this domain cannot contain $t=Q / 2$ for $b>1$ and $t=1$ for $b \leq 1$, which is the location of a termination point transition for the pinned fBm0 (it corresponds to events when the minimum is at $\theta_{m} \approx \theta_{A}$ ), analyzed in [19, 40. The domain should also be contained within $\operatorname{Im}(t)<1 / 2$ (for $\beta=2$ ) because of the integer nature of the field $\mathcal{N}(\theta)$. Extending the formula beyond remains open. However, results from [2, 65] for $\beta=2$, suggest that, treating $n b=-t$ and $b$ as independent variables, the integrand in $(20)$ can be extended formally to a sum over $b n \rightarrow b n+i \mathbb{Z}, b \rightarrow b+i \mathbb{Z}$. Investigating these properties is left for future studies.

\section{MOMENTS OF THE POSITION OF THE MAXIMUM}

As to the position of the maximum $\theta_{m} \in\left[\theta_{A}, \theta_{B}\right]$, we recall that its statistics for the $\mathrm{fBm} 0$ on an interval has been investigated in [20] by calculating those of the Jacobi ensemble of random matrices and performing the continuation to $n=0$. Defining $y_{m}=\left(\theta_{m}-\theta_{A}\right) / \ell$, the moments $\mathbb{E}\left(y_{m}^{k}\right)$ are thus the ones given in [20] (in Eqs. (129-130) for $k=2,4$ and Eqs. (101),(98-100), and Appendix C for general $k$ ). Extending that calculation to treat the case of the mesoscopic interval, one checks that the additional factors in (54) do not contribute, and arrives at the results mentioned in the Letter.

\section{JOINT MOMENTS OF THE POSITION AND THE VALUE OF THE MAXIMUM}

Preliminary remark. Consider two random variables $X_{1}$ and $X_{2}$. By definition the connected moments (also called bivariate cumulants) are given by

$$
\mathbb{E}_{c}\left(X_{1}^{q_{1}} X_{2}^{q_{2}}\right)=\left.\left.\partial_{t_{1}}^{q_{1}}\right|_{t_{1}=0} \partial_{t_{2}}^{q_{2}}\right|_{t_{2}=0} \log \mathbb{E}\left(e^{t_{1} X_{1}+t_{2} X_{2}}\right)
$$

Let us define the following biased average

$$
\left\langle f\left(X_{2}\right)\right\rangle_{t_{1}}=\frac{\mathbb{E}\left(f\left(X_{2}\right) e^{t_{1} X_{1}}\right)}{\mathbb{E}\left(e^{t_{1} X_{1}}\right)}
$$

Expanding the r.h.s of 61 in powers of $t_{2}$ we see that

$$
\mathbb{E}_{c}\left(X_{2} X_{1}^{q}\right)=\left.\partial_{t_{1}}^{q}\right|_{t_{1}=0}\left\langle X_{2}\right\rangle_{t_{1}} \quad, \quad \mathbb{E}_{c}\left(X_{2}^{2} X_{1}^{q}\right)=\left.\partial_{t_{1}}^{q}\right|_{t_{1}=0}\left(\left\langle X_{2}^{2}\right\rangle_{t_{1}}-\left\langle X_{2}\right\rangle_{t_{1}}^{2}\right)
$$

and so on, which is also equivalent (upon multiplying by $1 / q$ ! and summing over $q$ ) to the following formula for the generating functions of the bivariate cumulants of lowest order in $X_{2}$

$$
\mathbb{E}_{c}\left(X_{2} e^{t_{1} X_{1}}\right)=\left\langle X_{2}\right\rangle_{t_{1}}=\frac{\mathbb{E}\left(X_{2} e^{t_{1} X_{1}}\right)}{\mathbb{E}\left(e^{t_{1} X_{1}}\right)} \quad, \quad \mathbb{E}_{c}\left(X_{2}^{2} e^{t_{1} X_{1}}\right)=\left\langle X_{2}^{2}\right\rangle_{t_{1}}-\left\langle X_{2}\right\rangle_{t_{1}}^{2}
$$

which will be useful below. 


\section{Mesoscopic interval}

Let us discuss first the mesoscopic interval. Let us denote, as in the text, $y=\frac{\theta-\theta_{A}}{\ell} \in[0,1]$, and $\left\langle y^{k}\right\rangle$ the $k$-th moment of the random variable $y$ with respect to the Gibbs measure associated to $Z_{b}$ defined in (14), for a fixed random configuration of the eigenvalues $\theta_{i}$. One can evaluate the following ratio of averages w.r.t. the measure $\mathrm{CUE}_{\beta}$ for the eigenvalues

$$
\frac{\mathbb{E}\left[\left\langle y^{k}\right\rangle Z_{b}^{n}\right]}{\mathbb{E}\left[Z_{b}^{n}\right]}=\left.\left\langle y^{k}\right\rangle_{\beta, a, b, n}\right|_{(\beta, a, b) \rightarrow\left(b, 2 n b^{2}, 0\right)}=M_{k}(t=-b n, b)
$$

The numerator in the l.h.s. of (65) equals Eq. (54) of the text with $x_{a} \rightarrow y_{a}$ and $y_{1}^{k}$ inserted in the integrand. The corresponding ratio is thus the $k$-th moment of the Jacobi ensemble denoted $\left\langle y^{k}\right\rangle_{\beta, a, b, n}$ in [20] with the identification of parameters corresponding to $\mathrm{fBm} 0$ (see Eqs. $(56,57)$ there). Note that the extra factors containing $A_{\beta}(z)$ in $(54)$, not present in the fBm0, drop out in the ratio. The expression for the $\left\langle y^{k}\right\rangle_{\beta, a, b, n}$ were obtained in [20] and we denote $M_{k}(t=-b n, b)$ these expressions, which are rational fractions of the variables $t=-b n$ and $b$. We thus obtain

$$
\mathbb{E}\left(\left\langle y^{k}\right\rangle e^{-2 \pi \sqrt{\frac{\beta}{2}} t \mathcal{F}}\right)=M_{k}(t, b) \mathbb{E}\left(e^{-2 \pi \sqrt{\frac{\beta}{2}} t \mathcal{F}}\right)
$$

which is valid in the high temperature phase $b<1$. The simplest examples are the first two moments $k=1,2$. From (107) and (190) in [20] we obtain

$$
M_{1}(t, b)=\frac{1}{2}-\frac{t b}{2\left(1+b^{2}\right)} \quad, \quad M_{2}(t, b)=\frac{\left(b^{2}-b t+1\right)\left(b\left(b\left(4 b^{2}-b t+t^{2}+9\right)-t\right)+4\right)}{2\left(6 b^{6}+19 b^{4}+19 b^{2}+6\right)}
$$

These expressions are duality invariant, i.e. does not change under $b \rightarrow 1 / b$. All moments $M_{k}(t, b)$ share this property [20] (their explicit expressions are given in (91-92) there). Hence the freezing duality conjecture (FDC) allows to continue (66) for $b>1$. As in the text, the r.h.s. is duality invariant if multiplied by $\Gamma\left(1+\frac{t}{b}\right)$, hence the value of the l.h.s, as a function of $b$, freezes at $b=1$. Taking $b \rightarrow+\infty$ we obtain

$$
\mathbb{E}\left(y_{m}^{k} e^{-2 \pi \sqrt{\frac{\beta}{2}} t \delta \mathcal{N}_{m}}\right)=M_{k}(t, 1) \mathbb{E}\left(e^{-2 \pi \sqrt{\frac{\beta}{2}} t \delta \mathcal{N}_{m}}\right)
$$

where $y_{m}$ is the position of the maximum. Setting $t=0$ yields the results for the moments $\mathbb{E}\left(y_{m}^{k}\right)$ quoted in the text. Let us denote the centered variables

$$
\tilde{y}_{m}=y_{m}-\mathbb{E}\left(y_{m}\right) \quad, \quad \delta \tilde{\mathcal{N}}_{m}=\delta \mathcal{N}_{m}-\mathbb{E}\left(\delta \mathcal{N}_{m}\right)
$$

Consider 68) for $k=1$. Using that $\mathbb{E}\left(y_{m}\right)=\frac{1}{2}$, this can be written as

$$
\mathbb{E}\left(\tilde{y}_{m} e^{-2 \pi \sqrt{\frac{\beta}{2}} t \delta \tilde{\mathcal{N}}_{m}}\right)=-\frac{t}{4} \mathbb{E}\left(e^{-2 \pi \sqrt{\frac{\beta}{2}} t \delta \tilde{\mathcal{N}}_{m}}\right) \quad \Leftrightarrow \quad\left\langle\tilde{y}_{m}\right\rangle_{t}=-\frac{t}{4}
$$

where it is useful to define the following averages

$$
\left\langle f\left(\tilde{y}_{m}\right)\right\rangle_{t}:=\frac{\mathbb{E}\left(f\left(\tilde{y}_{m}\right) e^{-2 \pi \sqrt{\frac{\beta}{2}} t \delta \tilde{\mathcal{N}}_{m}}\right)}{\mathbb{E}\left(e^{-2 \pi \sqrt{\frac{\beta}{2}} t \delta \tilde{\mathcal{N}}_{m}}\right)}
$$

which represent averages under a biased probability $e^{-2 \pi \sqrt{\frac{\beta}{2}} t \delta \tilde{\mathcal{N}}_{m}} \times \mathcal{P}\left(\delta \tilde{\mathcal{N}}_{m}\right)$, where $\mathcal{P}$ is the PDF of $\delta \tilde{\mathcal{N}}_{m}$. Here $t<0$ corresponds to biasing the values of the maximum towards the positive values and leads to positive values on average for $\tilde{y}_{m}$. Expansion in powers of $t$ in 70 yields the relations, valid for any $p \geq 0$

$$
\mathbb{E}\left(\tilde{y}_{m}\left(\delta \tilde{\mathcal{N}}_{m}\right)^{p}\right)=\frac{p}{4} \frac{1}{2 \pi \sqrt{\beta / 2}} \mathbb{E}\left(\left(\delta \tilde{\mathcal{N}}_{m}\right)^{p-1}\right)=p \mathbb{E}\left(\tilde{y}_{m} \delta \tilde{\mathcal{N}}_{m}\right) \times \mathbb{E}\left(\left(\delta \tilde{\mathcal{N}}_{m}\right)^{p-1}\right)
$$

In particular

$$
\mathbb{E}\left(\tilde{y}_{m}\left(\delta \tilde{\mathcal{N}}_{m}\right)^{p}\right)=\frac{1}{(2 \pi \sqrt{\beta / 2})^{p}} \times\left\{\begin{array}{l}
\frac{1}{4} \quad, \quad p=1 \\
0 \quad, \quad p=2 \\
\frac{3}{4}\left(2 \log N \ell+\frac{9}{4}\right), \quad p=3 \\
\left(-\frac{17}{4}+\frac{8 \pi^{2}}{3}-8 \zeta(3)\right) \quad, \quad p=4
\end{array}\right.
$$

The result for $p=1$ shows that positions of maximum $y_{m}>1 / 2$ correlate with values of the maximum larger than the average, consistent with the pinning at $y=0$, i.e. $\delta \mathcal{N}\left(\theta=\theta_{A}\right)=0$, while the boundary condition at $y=1$ is free. Since $\delta \mathcal{N}_{m}-\mathbb{E}\left(\delta \mathcal{N}_{m}\right)$ is typically $\sim \sqrt{\log N \ell}$ the correlation with the Gaussian part of the fluctuations of the value of the maximum is absent in the correlation for $p=1$ (which is $O(1)$ ). Now, it is easy to see that 72 and 70 imply 
that all higher bi-variate cumulants vanish, i.e. the information contained in (70) can be summarized as

$$
\begin{aligned}
& \mathbb{E}\left(\tilde{y}_{m} \delta \tilde{\mathcal{N}}_{m}\right)=\frac{1}{4} \frac{1}{2 \pi \sqrt{\beta / 2}} \\
& \mathbb{E}_{c}\left(\tilde{y}_{m}\left(\delta \tilde{\mathcal{N}}_{m}\right)^{p}\right)=0 \quad, \quad p \geq 2
\end{aligned}
$$

consistent with 73 which is the sum of all disconnected averages.

For $k=2$ we obtain

$$
\mathbb{E}\left(y_{m}^{2} e^{-2 \pi \sqrt{\frac{\beta}{2}} t \delta \tilde{\mathcal{N}}_{m}}\right)=\frac{1}{100}(2-t)\left(17-2 t+t^{2}\right) \mathbb{E}\left(e^{-2 \pi \sqrt{\frac{\beta}{2}} t \delta \tilde{\mathcal{N}}_{m}}\right) \quad \Leftrightarrow \quad\left\langle y_{m}^{2}\right\rangle_{t}=\frac{1}{100}(2-t)\left(17-2 t+t^{2}\right)
$$

For $t=0$ we obtain the result given in the text $\mathbb{E}\left(y_{m}^{2}\right)=\frac{17}{50}$. Expansion of the first equation in powers of $t$ allows to obtain all joint moments of the form $\mathbb{E}\left(y_{m}^{2}\left(\delta \tilde{\mathcal{N}}_{m}\right)^{p}\right)$ using our results for the cumulants of the value of the maximum (given in the text). Alternatively we may write the bi-variate cumulants (see preliminary remark above)

$$
\mathbb{E}_{c}\left(y_{m}^{2} e^{-2 \pi \sqrt{\frac{\beta}{2}} t \delta \tilde{\mathcal{N}}_{m}}\right)=\left\langle y_{m}^{2}\right\rangle_{t}-\left\langle y_{m}\right\rangle_{t}^{2}=\frac{1}{400}\left(4-t^{2}\right)(9+4 t)
$$

Expanding in powers of $t$ on both sides we see that for $k=2$, only the first three connected moments are non zero.

\section{Full circle}

Consider now the average of $\cos k \phi$ with respect to the Gibbs measure associated to $Z_{b}$, defined in (14), on the full circle, i.e. with $\theta_{A}=-\pi$ and $\theta_{B}=\pi$. Again one can evaluate the ratio of averages w.r.t. the measure CUE $\mathrm{E}_{\beta}$ for the eigenvalues

$$
\frac{\mathbb{E}\left[\langle\cos k \phi\rangle Z_{b}^{n}\right]}{\mathbb{E}\left[Z_{b}^{n}\right]}=\langle\cos (k \phi)\rangle_{\beta, \mu, n}=\left.(-1)^{k}\left\langle y^{k}\right\rangle_{\beta, a, b, n}\right|_{(\beta, a, b) \rightarrow\left(b,-1-b^{2}, 2 n b^{2}\right)}=\tilde{M}_{k}(t=-b n, b)
$$

The numerator in the l.h.s. of (78) equals Eq. (20) of the text with $\cos k \phi_{1}$ inserted in the integrand. The second equality is the conjecture obtained in Eqs. (157-158) in 20] (where $\langle\cos (k \phi)\rangle_{\beta, \mu, n}$ denotes the 1.h.s. in (158) there, with $\kappa=-\beta^{2} \rightarrow-b^{2}$ and $\mu \rightarrow n b^{2}$ ) which relates the moments in the circular ensemble to those on the interval, i.e the moments $\left\langle y^{k}\right\rangle_{\beta, a, b, n}$ already used in the previous section. The different specialisations of the parameters leads to other rational functions $\tilde{M}_{k}(t=-b n, b)$. From (107) and (91-92) in [20] we obtain

$$
\tilde{M}_{1}(t, b)=-\frac{t b}{1+b^{2}} \quad, \quad \tilde{M}_{2}(t, b)=\frac{b t\left(b^{4}+2 b^{3} t-b^{2} t^{2}+3 b^{2}+2 b t+1\right)}{\left(b^{2}+1\right)\left(b^{2}+2\right)\left(2 b^{2}+1\right)}
$$

These expressions are again duality invariant. From the FDC we obtain

$$
\mathbb{E}\left(\cos \left(k \theta_{m}\right) e^{-2 \pi \sqrt{\frac{\beta}{2}} t \delta \mathcal{N}_{m}}\right)=\tilde{M}_{k}(t, 1) \mathbb{E}\left(e^{-2 \pi \sqrt{\frac{\beta}{2}} t \delta \mathcal{N}_{m}}\right)
$$

where $\theta_{m}$ is the position of the maximum of $\delta \mathcal{N}(\theta)$ on the full circle. We can check that for $t=0$ all $\mathbb{E}\left(\cos \left(k \theta_{m}\right)\right)=0$, $\theta_{m}$ has a uniform distribution on the circle. For $k=1$ we obtain

$$
\mathbb{E}\left(\cos \left(\theta_{m}\right) \delta \tilde{\mathcal{N}}_{m}\right)=\frac{1}{2} \frac{1}{2 \pi \sqrt{\beta / 2}}
$$

which shows that higher values of the maximum correlate with $\cos \left(\theta_{m}\right)>0$, i.e. $\theta_{m}$ being closer to 0 than to $\pm \pi$, the point at which its value is pinned to zero. Again we see that all connected correlations $\mathbb{E}_{c}\left(\cos \left(\theta_{m}\right)\left(\delta \tilde{\mathcal{N}}_{m}\right)^{p}\right)$ vanish. For $k=2$ we obtain

$$
\mathbb{E}\left(\cos \left(2 \theta_{m}\right) e^{-2 \pi \sqrt{\frac{\beta}{2}} t \delta \tilde{\mathcal{N}}_{m}}\right)=\frac{1}{18}(5-t) t(t+1) \mathbb{E}\left(e^{-2 \pi \sqrt{\frac{\beta}{2}} t \delta \tilde{\mathcal{N}}_{m}}\right)
$$

which, upon expanding in $t$ yields all joint moments of the form $\mathbb{E}\left(\cos \left(2 \theta_{m}\right)\left(\delta \tilde{\mathcal{N}}_{m}\right)^{p}\right)$. 